\title{
Optimal Sensor Placement for a Constellation of Multistatic Narrowband Pixelated Sensors
}

\author{
Chris Kreucher
}

\begin{abstract}
This paper presents a novel approach to selecting the location of a constellation of multistatic RF sensors to optimize system detection and tracking. The method works by computing the posterior Cramér-Rao lower bound on localization error as a function of sensor positions, and then selecting sensor locations to minimize the bound. One important contribution of this study is the derivation of the bound for a non-Gaussian, nonlinear pixelated sensor model which includes transmit and receive beam patterns, direct path energy, and target impulse response. A second contribution is a report on the results of two field collections which illustrate the efficacy of the method.
\end{abstract}

Index Terms-Cramér-Rao bound, estimation, RF, resource management, sensor placement, tracking.

\section{INTRODUCTION}

$\mathbf{T}$ HIS paper describes a novel method to automatically place a constellation of multistatic narrowband RF sensors, and illustrates the efficacy of the system with two field experiments. The approach is to compute the posterior Cramér-Rao lower bound (PCRLB) on estimation error as a function of sensor position, and then select sensor locations which minimize a utility function that is based on the bound. The main contributions of this paper are the derivation of the bound for a nonlinear/nonGaussian pixelated sensor model and an illustration of the technique with two field experiments. The derivation takes into account a number of physical factors, including antenna beam patterns and target impulse response. The experiments provide empirical evidence as to the efficacy of the technique.

In the multistatic pulsed radar system which we consider here, the physics of the received data are not well approximated by a traditional linear/Gaussian model. To accurately approximate the received data, we must model the non-Gaussian background and target statistics, the nonlinear measurement to target state coupling, as well as the effect of transmit and receive beam patterns. The PCRLB method which is presented here uses such a model and is thus tailored to the pixelated nature of the sensors, antenna beam patterns, and the non-Gaussian measurement statistics. As such, it automatically favors sensor placements that yield beneficial multistatic spatial diversity, placements that

Manuscript received March 4, 2011; revised July 27, 2011 and November 23, 2011; accepted February 1, 2012. Date of publication March 19, 2012; date of current version December 17, 2012. This work was supported by the Air Force Research Laboratory under Contract FA8650-09-M-1549 and Contract FA8650-10-C-1718. This paper was recommended by Associate Editor Y. Jin.

The author is with the Fusion Group, Integrity Applications Inc., Ann Arbor, MI 48108 USA (e-mail: ckreuche @ umich.edu).

Color versions of one or more of the figures in this paper are available online at http://ieeexplore.ieee.org.

Digital Object Identifier 10.1109/TSMCC.2012.2187187 illuminate the important portions of the surveillance region, and placements that have appropriate receive beams.

A system which is based on a constellation of narrowband sensors has a number of benefits over a single wideband sensor in this application. First, commercial applications have led to an erosion of the available spectrum meaning often that only a small portion is available for other use [1], [2]. Furthermore, narrowband sensors are inexpensive due to their simple electronics, they require low energy consumption, they are easy to maintain, and it is easy to communicate their data to a centralized processing point. However perhaps most importantly, a constellation of narrowband sensors provides geometric diversity. By exploiting multistatic returns using advanced signal-processing techniques, one can trade costly spectral diversity for cost-efficient spatial diversity, and provide performance improvement.

Tharmarasa's work [3], [4], which appeared recently in this journal, breaks sensor management into two disciplines: sensor placement and sensor selection. The authors of [3] deal with optimal sensor selection. Our study is complimentary in that it deals with the other issue, optimal sensor placement.

There is other literature which investigates CRLB methods for optimal sensor placement. For example, in [5] sensor placement is considered using the CRLB to estimate range and bearing when sensors directly measure time difference of arrival. Given their linear and Gaussian noise model and the physical assumption that sensors are closely spaced and distant from the target, the authors of [5] are able to analytically derive locations that minimize the CRLB on range and bearing. Early work from [6]-[8] considered similar scenarios that are applicable to the passive sonar problem, but restricted sensor positions to being along a line. These efforts find a interestingly simple optimal sensor layout under the linear assumption. In [9], an approach to sensor placement is presented for active sonar that uses a weighted Fisher information matrix as the objective function. It applies a minimax sensor placement strategy using the model of multistatic returns and error covariance matrices from [10]. Finally, in [11] the PCRLB is used to develop a method to place sensors. Target measurements are modeled as threshold exceedances related nonlinearly to the target state embedded in Gaussian noise. The derivation treats both the measurement origin of uncertainty and false alarm issue.

In contrast with these earlier works, in our approach we model sensor measurements as pixelated Rayleigh returns. This nonthresholded, nonlinear, non-Gaussian sensor model more accurately describes the multistatic RF measurements of our application, and leads to a very different expression.

This paper proceeds as follows. First, Section II introduces the multistatic range/range-rate statistical model and the factors 


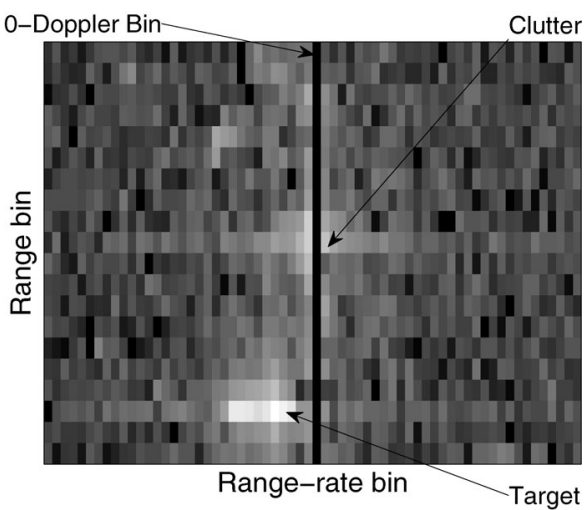

Fig. 1. Bistatic range/range-rate image with a single target.

that influence the received data. Section III describes the sensor placement algorithm. The explicit derivations are relegated to the Appendices. Section IV gives experimental results that validate the method. Finally, Section V concludes.

\section{Sensor Statistical Modeling}

We assume a constellation of $N$ sensors. To simplify notation, we assume that each sensor is both a transmitter and a receiver, although this is not required by the approach. A sensor transmits a narrowband RF signal, which is reflected off the target and received at each sensor. This process repeats $N$ times, with each sensor serving as a transmitter. There are then data from $N^{2}$ sensor pairs at each time. For each sensor pair, Fourier processing on the received data generates a bistatic range/rangerate surface [12] like that shown in Fig. 1.

These range/range-rate surfaces are related to the state of the target nonlinearly, and are corrupted by various types of noise. The aim of this section is to describe a physics-based statistical model of the observables.

Let $z_{i j}$ denote the envelope-detected value in the $(i, j)$ th bistatic range/range-rate resolution bin (cell). The number of cells and the cell resolution are determined by the number of pulses, the pulse repetition frequency (PRF), the coherent pulse interval (CPI), and the bandwidth of the radar [13]. For example, the bistatic range resolution is given by $c / \mathrm{BW}$, where $c$ is the speed of light, and BW is the radar bandwidth. Similarly, the bistatic range-rate resolution is given by $c / f_{c} T$, where $f_{c}$ is the radar center frequency, and $T$ is the CPI. The collection of measurements for a particular transmitter/receiver pair is then the matrix of bistatic range/range-rate correlations in each cell, i.e., the matrix

$$
z=\left(\begin{array}{ccc}
z_{11} & \cdots & z_{1 N_{D}} \\
& \cdots & \\
z_{N_{r} 1} & \cdots & z_{N_{r} N_{D}}
\end{array}\right)
$$

where $N_{R}$ and $N_{D}$ are the number of bistatic range and rangerate cells, respectively.

Let the vector $\theta=[x, \dot{x}, y, \dot{y}]$ describe the true position and velocity of the target, and $x_{T}(t), y_{T}(t), x_{R}(r), y_{R}(r)$ define the location of a transmitter $t$ and a receiver $r$. The statistics of the observation $z_{i j}(t, r)$ in the cell $(i, j)$ from the transmitter $t$ and the receiver $r$ depend on a number of physical factors: The following which are modeled here. The proximity of cell $(i, j)$ to the true bistatic range and range-rate of the target as measured from a transmitter at $t$ and a receiver at $r$; where the target is in the illumination beam of the transmitter; where the target is in the receive bin of the receiver; and whether the receiver is saturated with direct-path energy from the transmitter. Given the small size of the surveillance region, in this study we have ignored range-dependent (signal-to-noise ratio) SNR effects. In addition, given the mild aspect-dependent scattering of our targets, we ignore aspect-dependent target scattering. Additional effects such as these could be incorporated into the framework below in applications where they are relevant without significantly affecting model complexity.

We model the statistics in each bistatic range/range-rate cell as Rayleigh. Let $f_{i j}\left(z_{i j} ; \theta, t, r\right)$ denote the probability distribution function for the received energy in the bistatic range/range-rate cell $(i, j)$ given transmitter $t$, receiver $r$, and a target at $\theta$. The statistical model is then

$$
f_{i j}\left(z_{i j} ; \theta, t, r\right)=2 \frac{z_{i j}}{\lambda_{i j}^{2}(\theta ; t, r)} \exp \left(-\frac{z_{i j}^{2}}{\lambda_{i j}^{2}(\theta ; t, r)}\right)
$$

where $\lambda_{i j}(\theta ; t, r)$ is the pixel-dependent Rayleigh mode parameter defined later which accounts for the physical factors by specifying the energy expected to be received in the cell $(i, j)$.

We assume that noise is independent across pixels and write the likelihood for the measurement matrix as

$$
f(z ; \theta, t, r)=\prod_{i, j} 2 \frac{z_{i j}}{\lambda_{i j}^{2}(\theta ; t, r)} \exp \left(-\frac{z_{i j}^{2}}{\lambda_{i j}^{2}(\theta ; t, r)}\right)
$$

and with multiple transmitters and receivers write

$$
f(z ; \theta)=\prod_{t, r} \prod_{i, j} 2 \frac{z_{i j}(t, r)}{\lambda_{i j}^{2}(\theta ; t, r)} \exp \left(-\frac{z_{i j}^{2}(t, r)}{\lambda_{i j}^{2}(\theta ; t, r)}\right) .
$$

The notation $\prod_{t, r}$ is to be interpreted as specifying a product over all transmit/receive pairs, and $z$ is now expanded to mean the collection of all measurements from all transmit/receive pairs.

We now codify precisely how the Rayleigh mode parameter depends on the physical factors that are outlined previously. Our model is

$\lambda_{i j}(\theta ; t, r)$

$$
=\lambda_{B}(t, r)+\left(\lambda_{T}(t, r)-\lambda_{B}(t, r)\right) \prod_{f=1}^{4} S_{i j}^{f}(\theta ; t, r)
$$

where $\lambda_{B}(t, r)$ and $\lambda_{T}(t, r)$ are the background and target mode parameters, and $S_{i j}^{f}(\theta ; t, r)$ model the SNR suppression from the physical factors outlined above on pixel $i j$.

The $S^{f}$ which we have chosen to include in our model are defined as follows. In the case of directional antennas, we must model the transmit and receive beam patterns. Let the angles $\alpha_{t}$ and $\alpha_{r}$ represent the transmitter and receiver pointing angles, respectively. Let $\alpha_{t, \theta}$ be the angle between the transmitter and 
the target. Define $\alpha_{r, \theta}$ and $\alpha_{t, r}$ analogously, i.e.,

$$
\begin{aligned}
\alpha_{t, \theta} & =\arctan \left(\frac{y-y_{T}(t)}{x-x_{T}(t)}\right) \\
\alpha_{r, \theta} & =\arctan \left(\frac{y-y_{R}(r)}{x-x_{R}(r)}\right) \\
\alpha_{t, r} & =\alpha_{t}-\alpha_{r} .
\end{aligned}
$$

With this as background, $S_{i j}^{1}(\theta ; t, r)$ will model the SNR suppression of the transmit illumination beam, taken to be a sinc-squared [14] with beamwidth controlled by the constant $c$ (specified by the particular antenna hardware):

$$
S_{i j}^{1}(\theta ; t, r)=\operatorname{sinc}^{2}\left(c\left(\alpha_{t}-\alpha_{t, \theta}\right)\right) .
$$

As the target moves from the center of the illumination beam, this factor depresses the ability to discriminate the target from the background.

Likewise, $S_{i j}^{2}(\theta ; t, r)$ will model the receive beam

$$
S_{i j}^{2}(\theta ; t, r)=\operatorname{sinc}^{2}\left(c\left(\alpha_{r}-\alpha_{r, \theta}\right)\right) .
$$

In the case of omnidirectional antennas, $S^{1}$ and $S^{2}$ are omitted from the computation (or, alternatively $c=0$ ). Notice that these suppression terms do not change with pixel $i j$, but we have included the subscripts for notational convenience.

Next, in order to capture the proximity of cell $(i, j)$ to the true range/range-rate values, let

$$
\Delta_{i j}(\theta ; t, r)=\left(\begin{array}{c}
h_{i j}^{R}-R(\theta ; t, r) \\
h_{i j}^{\dot{R}}-\dot{R}(\theta ; t, r)
\end{array}\right)
$$

where $R(\theta ; t, r)$ is the function that maps the target state $\theta$ to a bistatic range, and $\dot{R}(\theta ; t, r)$ is the function that maps the target state $\theta$ to a bistatic range-rate, i.e.,

$$
\begin{aligned}
R(\theta ; t, r)= & \sqrt{\left(x-x_{R}(r)\right)^{2}+\left(y-y_{R}(r)\right)^{2}} \\
& +\sqrt{\left(x-x_{T}(t)\right)^{2}+\left(y-y_{T}(t)\right)^{2}}
\end{aligned}
$$

and

$$
\begin{aligned}
\dot{R}(\theta ; t, r)= & \frac{\left(x-x_{R}(r)\right) \dot{x}+\left(y-y_{R}(r)\right) \dot{y}}{\sqrt{\left(x-x_{R}(r)\right)^{2}+\left(y-y_{R}(r)\right)^{2}}} \\
& +\frac{\left(x-x_{T}(t)\right) \dot{x}+\left(y-y_{T}(t)\right) \dot{y}}{\sqrt{\left(x-x_{T}(t)\right)^{2}+\left(y-y_{T}(t)\right)^{2}}} .
\end{aligned}
$$

$h_{i j}^{R}$ and $h_{i j}^{\dot{R}}$ are the functions that map $(i, j)$ to bistatic range and bistatic range-rate, respectively. Furthermore, $R$ and $\dot{R}$ are wrapped at the range and range-rate ambiguities.

With these definitions, $S_{i j}^{3}(\theta ; t, r)$ will model the target impulse response. Here, we have chosen to simply model the mainlobe of the response and use

$$
S_{i j}^{3}(\theta ; t, r)=\exp \left(-\Delta_{i j}^{T} A \Delta_{i j}\right)
$$

where $A$ specifies the range and range-rate extent of the mainlobe and provides the coupling between the two.
The final term models the effect of direct path energy on the ability to discriminate bistatic target returns. If the direct path between the transmitter and the receiver puts energy in the target range bin, this depresses the ability to discriminate the target. This factor will be denoted by $S_{i j}^{4}(\theta ; t, r)$. A straightforward but useful model to reflect the SNR degradation that direct path energy will create is

$$
S_{i j}^{4}(\theta ; t, r)=1-\exp \left(\frac{-\left(h_{i j}^{R}-R_{t, r}\right)^{2}}{2 \sigma_{d}^{2}}\right)
$$

where $R_{t, r}$ is range between transmitter $t$ and receiver $r$.

Taken together, (4), (5), and the definitions of $S_{i j}^{f}(\theta ; t, r)$ completely specify the sensor model, which says that pixels in the range/range-rate map have Raleigh statistics with the mode parameter determined by how close the range/range-rate value represented by the pixel is to the targets' true bistatic range/rangerate, the direct path energy, and beam-pattern-induced factors.

\section{POSTERIOR CRAMÉR-RAO LOWER BOUND FOR SENSOR PLACEMENT}

The sensor placement algorithm that we propose selects sensor positions to yield the best performance in estimating target location in terms of the PCRLB. In other words, we choose sensor positions to yield the best lower bound on achievable estimator performance, as measured by the PCRLB. We focus on a tracking context, where we estimate a target state which evolves over time.

There are other bounds that could be applied in this context. These include the Bayesian Bhattacharyya bound [15], the Bobrovsky-Zakai bound [16], the Weiss-Weinstein bound [17], [18], and the so-called combined Bayesian bounds [19]. In some cases, these methods provide tighter bounds on estimator performance. We have chosen the PCRLB, as others typically do [20], in part because there exists a computationally efficient recursive form of the bound.

For any estimator $\hat{\theta}(z)$, the PCRLB on the estimation error [20] has the form

$$
\mathbb{E}_{z, \theta}\left[(\hat{\theta}(z)-\theta)(\hat{\theta}(z)-\theta)^{T}\right] \geq J_{B}^{-1}
$$

where $J_{B}$ is known as Bayesian information matrix (BIM). Its $(m, n)$ th element is given by

$$
\left[J_{B}\right]_{m n}=\mathbb{E}_{z, \theta}\left[\frac{\partial \ln p(z, \theta)}{\partial \theta_{m}} \cdot \frac{\partial \ln p(z, \theta)}{\partial \theta_{n}}\right] .
$$

Let us denote the Fisher information matrix (FIM) as

$$
\left[J_{F}(\theta)\right]_{m n}=\mathbb{E}_{z}\left[\frac{\partial \ln p(z \mid \theta)}{\partial \theta_{m}} \cdot \frac{\partial \ln p(z \mid \theta)}{\partial \theta_{n}}\right]
$$

and define its expectation with respect to $\theta$ as $J_{D}$, i.e.,

$$
\left[J_{D}\right]_{m n}=\mathbb{E}_{\theta}\left[\left[J_{F}(\theta)\right]_{m n}\right] .
$$

Finally, define the prior information matrix as

$$
\left[J_{p}\right]_{m n}=\mathbb{E}_{\theta}\left[\frac{\partial \ln p(\theta)}{\partial \theta_{m}} \cdot \frac{\partial \ln p(\theta)}{\partial \theta_{n}}\right] .
$$

Then, the BIM can be written [20] as the sum of the information matrix from the data and the information matrix from prior 
information, i.e.,

$$
J_{B}=J_{D}+J_{P} .
$$

Our first step is to derive $J_{F}$ for the sensor model in Section II. Defining $p(z \mid \theta)$ from the model of (4) and the definitions in (9)-(15), Appendix A [21] shows through a straightforward but lengthy derivation that under this model the $(m, n)$ th element of the FIM is

$$
\begin{aligned}
& {\left[J_{F}(\theta)\right]_{m n}=16 \sum_{t, r} \sum_{i, j} \frac{1}{\lambda_{i j t r}^{\theta 2}} \frac{\partial \lambda_{i j t r}^{\theta}}{\partial \theta_{m}} \frac{\partial \lambda_{i j t r}^{\theta}}{\partial \theta_{n}}} \\
& \quad+4\left(\sum_{t, r} \sum_{i, j} \frac{1}{\lambda_{i j t r}^{\theta}} \frac{\partial \lambda_{i j t r}^{\theta}}{\partial \theta_{m}}\right)\left(\sum_{p, q} \sum_{u, v} \frac{1}{\lambda_{u v p q}^{\theta}} \frac{\partial \lambda_{u v p q}^{\theta}}{\partial \theta_{n}}\right)
\end{aligned}
$$

where $\lambda_{i j t r}^{\theta} \doteq \lambda_{i j}(\theta ; t, r)$ is shorthand, and the summation $\sum_{t, r}$ is to be interpreted as summing over all transmit/receive pairs. To completely specify the terms in (22), we need the partial derivatives of the Rayleigh mode parameter defined in (5). From the chain rule, we have

$$
\begin{aligned}
& \frac{\partial \lambda_{i j}(\theta ; t, r)}{\partial \theta_{\circ}} \\
& \quad=\left(\lambda_{T}(t, r)-\lambda_{B}(t, r)\right) \sum_{f=1}^{4} \frac{\partial S_{i j}^{f}(\theta ; t, r)}{\partial \theta_{\circ}} \prod_{\substack{g=1 \\
g \neq f}}^{4} S_{i j}^{g}(\theta ; t, r)
\end{aligned}
$$

meaning that we need only to specify the partial derivatives of the individual terms $S^{f}$. Appendix B provides the analytic form of the partials of each of the model terms $S^{f}(\theta ; t, r)$ with respect to the state parameter $\theta$. With these calculations, the FIM (22) is fully specified.

$J_{D}(19)$ is the expectation of $J_{F}(\theta)$ with respect to $\theta$. This has been addressed in the literature [11] using Monte Carlo approximation, where $J_{F}$ is evaluated at many sample $\theta$ and averaged. We apply a similar approach, which is described in Section IV.

Given $J_{D}$, the BIM is computed as follows. In the tracking application where the target state evolves over time according to $\theta_{k+1}=g\left(\theta_{k}, w_{k}\right)$, Tichavsky et al. [22] provide a recursion giving the sequence of BIMs from $J_{D}$ at each time

$$
\begin{aligned}
& J_{B}(k+1) \\
& \quad=D_{k}^{33}-D_{k}^{21}\left(J_{B}(k)^{-1}+D_{k}^{11}\right)^{-1} D_{k}^{12}+J_{D}(k+1)
\end{aligned}
$$

where $D_{k}^{11}, D_{k}^{12}, D_{k}^{21}$, and $D_{k}^{33}$ depend on the model that is defined by the function $g$ [11]. The initial BIM $J_{B}(0)$ depends only on the prior $p(\theta)$. In the special case where the time evolution of the target state is modeled as $\theta_{k+1}=A \theta_{k}+w_{k}$, where $w_{k} \sim N(0, \Sigma)$, the Tichavsky recursion simplifies [11] to

$$
J_{B}(k+1)=\left(\Sigma+A J_{B}(k)^{-1} A^{T}\right)^{-1}+J_{D}(k+1) .
$$

Given the BIM, the corresponding PCRLB matrix $C$ is

$$
C_{k}=J_{B}(k)^{-1}
$$

and gives a lower bound on the performance of any estimator for this sensor model.

This is used as a metric to place the sensors as follows. For a candidate set of sensor locations $x_{1}, y_{1}, \ldots, x_{N}, y_{N}$, we compute $C_{k}$. We choose to use the average of the sum of the $x$ and $y$ error elements as a scalar metric for the utility of the constellation, i.e., the utility $U$ is

$$
U\left(x_{1}, y_{1}, \ldots, x_{N}, y_{N}\right)=\frac{1}{K} \sum_{k=1}^{K}\left(\left[C_{k}\right]_{x x}+\left[C_{k}\right]_{y y}\right)
$$

and propose to select the positions of the $N$ sensors as

$$
\hat{x}_{1}, \hat{y}_{1}, \ldots, \hat{x}_{N}, \hat{y}_{N}=\underset{x_{1}, \ldots, y_{N}}{\operatorname{argmin}} \frac{1}{K} \sum_{k=1}^{K}\left(\left[C_{k}\right]_{x x}+\left[C_{k}\right]_{y y}\right) .
$$

The utility $U$ is a function of $2 N$ variables ( $x$ and $y$ positions of the $N$ sensors), and as such optimal sensor placement requires optimization in $\mathbb{R}^{2 N}$. The surface is not convex; therefore, conventional optimization techniques which exploit convexity for efficient search through the solution space are not applicable. Techniques for global optimization of nonconvex multidimensional problems, including deterministic approaches such as branch-and-bound and heuristics-like decomposition [3], evolutionary algorithms [25], [29], memetic algorithms [27], [28], swarm methods [26], and stochastic methods [30], have received increased interest recently. In earlier work [21], we used an iterative-greedy heuristic with a related metric. In the iterativegreedy approach, all sensors are initially randomly placed. Then, the first sensor is replaced by holding the other sensors fixed and performing a single-sensor optimization. This is repeated for all sensors and iterated until no further movement occurs.

In the experiments that are described in Section IV, no formal optimization is necessary because we use the metric in the context of the location of a single sensor. The utility is then a function of only the $x$ and $y$ positions of the single sensor. In these experiments, the metric is evaluated over the 2-D position of a sensor by griding the state space in the region of feasible placements and evaluating the objective function at each point.

\section{RESUlTS}

This section describes two measurement campaigns that were performed to validate the PCRLB method described in this paper. The method was used both to predict estimation performance from arrangements of sensors and to place sensors. We then collected data with the sensors while a target was moving through the surveillance region. The collected data were processed with a Bayes-optimal tracker to estimate the target state. The tracking performance was compared with the PCRLB to validate the prediction. The PCRLB is a statistical bound; therefore, single tests are to be interpreted as validation of the method and not a rigorous verification. Since it is an integral part of the performance evaluation, a brief review of the tracker is provided in Section IV-A.

The collections used four RF antennas to measure information about range and range-rate of a moving dismount. They used a commercially available AKELA AVMU500A radar and SAS- 
510-4 (Yagi) antennas. The antennas are directional and are specified to have a $3 \mathrm{~dB}$ point of $41^{\circ}$ by the manufacturer. This is modeled explicitly in $S^{1}$ and $S^{2}$ [(9) and (10)]. Data were collected with $60 \mathrm{MHz}$ of bandwidth and a center frequency of $2.37 \mathrm{GHz}$, and used a $0.5 \mathrm{~s}$ CPI. This gave $5 \mathrm{~m}$ bistatic range resolution and $0.3 \mathrm{~m} / \mathrm{s}$ bistatic range-rate resolution. These parameters are encoded into the PCRLB model through $h^{R}$ and $h^{\dot{R}}$ of (11). Empirically, the impulse response has $\sigma_{R}=2.5 \mathrm{~m}$ and $\sigma_{\dot{R}}=0.6 \mathrm{~m} / \mathrm{s}$. These parameters are encoded into the matrix $A$ in (14). Calibration collections show that $\lambda_{B}=400$ and $\lambda_{T}=$ 500 are good estimates of the background and target modes for all sensor pairs, respectively. Taken together, these definitions completely specify the terms that are needed to compute the FIM [see (22)].

\section{A. Brief Overview of the Tracker}

In each field experiment, data from sensors were processed with a tracker to estimate the target state. This tracker estimate is compared with the PCRLB predictions to validate the approach. The tracker is described in [23], and is related to Kassas' approach, published recently in [24]. We provide a short overview here. The tracker is a Bayes-optimal nonlinear filter. It estimates the probability density on target state recursively and exploits both nonlinear and non-Gaussian measurement modalities without Kalman approximations. It does so by estimating $p(x \mid Z)$, the probability of the target state $x$ conditioned on all measurements made at all times by all sensors, $Z$. This is done using a Markov model of target state evolution to predict the density forward in time and a Bayes rule update to ingest new measurements. The target state evolution model is the linear Gaussian model that is discussed in Section III. The tracker is implemented using a multidimensional discrete grid.

\section{B. Collection Using the PCRLB to Place the Sensors}

This first experiment uses the PCRLB to select a transmitter location in a multistatic setup. We consider a surveillance application, where four sensors monitor traffic along a known road. We placed three sensors (antennas 2, 3, and 4), which will act as receivers only along the $y=0 \mathrm{~m}$ line at $x=0,10$, and $20 \mathrm{~m}$. We used the PCRLB method to compute the spatially varying utility to place the fourth sensor (the transmitter).

The computation of the utility $U$ for a particular sensor configuration $x_{1}, y_{1}, \ldots, x_{N}, y_{N}$ is performed as follows. First, we generated 250 example trajectories for a dismount walking the road. For this computation, the target state was discretized to $0.5 \mathrm{~s}$ CPI over a total of $30 \mathrm{~s}$ (i.e., 60 discrete points on the path). For each trajectory, we computed the FIM as a function of time $k$ using (22). Since we assumed a single transmitter and three receivers, only the terms corresponding to $t=3$ and $r=1,2,4$ are included. Computing the FIM (22) took about $0.7 \mathrm{~s}$ for each trajectory/proposed sensor location combination on a standard $2.8 \mathrm{GHz}$ Linux machine running unoptimized MATLAB code. (Note that this optimization will be performed offline one time and then sensors will be placed; therefore, computation time is not critical.) $J_{D}(k)$ was computed by averaging over the sample trajectories as an approximation to (19), and the BIM was com-

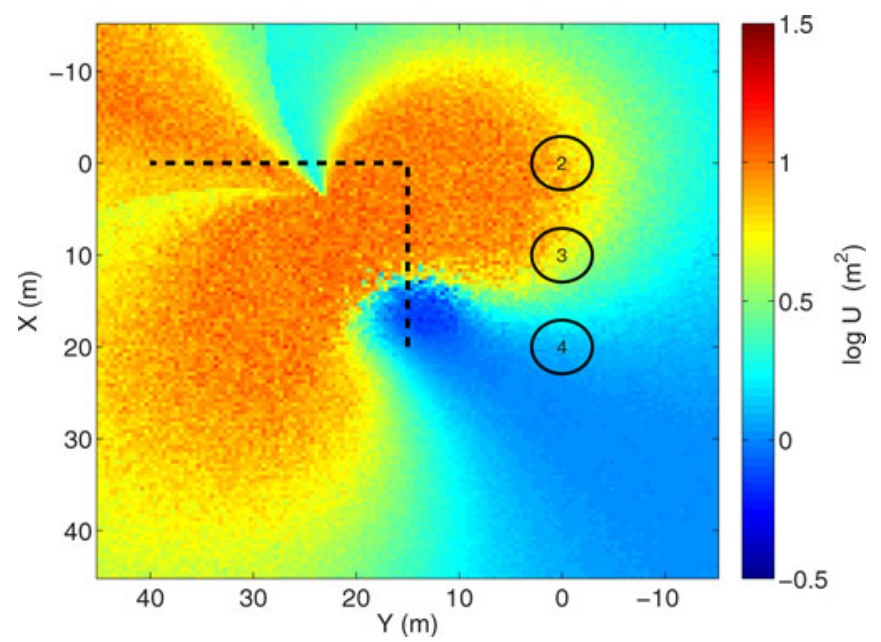

Fig. 2. PCRLB utility as a function of transmitter location. The average was taken over last 55 time steps of the path. We assumed receivers placed at $(0,0)$, $(10,0)$, and $(20,0)$, and computed (27) using the parameters given earlier for possible locations of a transmitter.

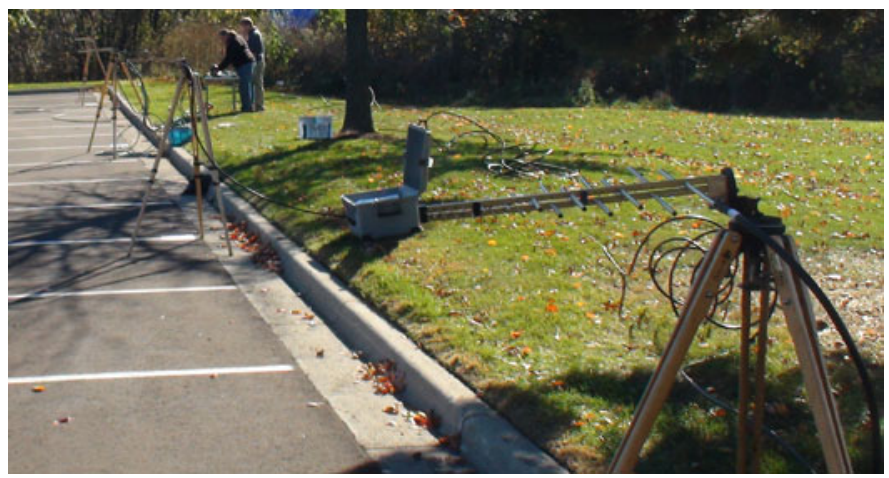

Fig. 3. Sensors as placed for configuration 1.

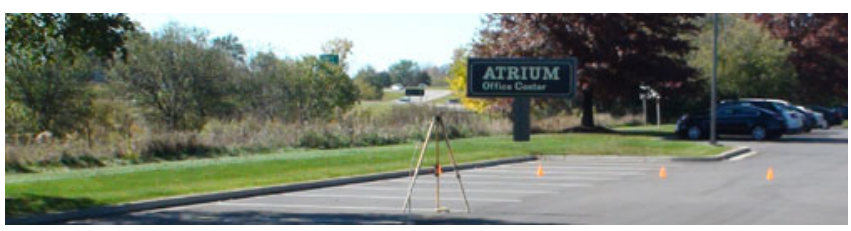

Fig. 4. Sensor 1 moved to collect data for configuration 2.

puted using the recursive relationship of (25). Next, the PCRLB as a function of time is given by (26). Finally, the utility $U$ as a function of transmitter location is computed using (27).

Fig. 2 shows the utility for a discrete set of potential transmitter locations.

We used this utility map to select two transmitter locations and collected data at each. The first configuration (see Fig. 3) placed the transmitter at a near-optimal location: $y=0 \mathrm{~m}$ and $x=35 \mathrm{~m}$. The second configuration (see Fig. 4) placed the transmitter at a suboptimal location: $y=30 \mathrm{~m}$ and $x=40 \mathrm{~m}$.

A dismount walked the road, while data were collected for each of two transmitter locations. Fig. 5 shows the setup. The path that was walked by the dismount, and the corresponding nominal CPI number (time) that the target was at each position, 


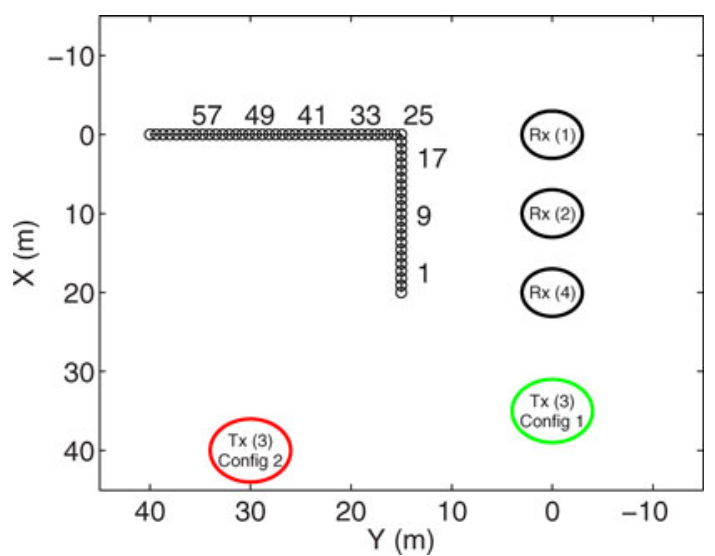

Fig. 5. View of the experiments from above, showing the road location, the three fixed receiver locations, and the two locations (configurations) for the transmitter. The nominal time steps corresponding to the dismount location are enumerated.

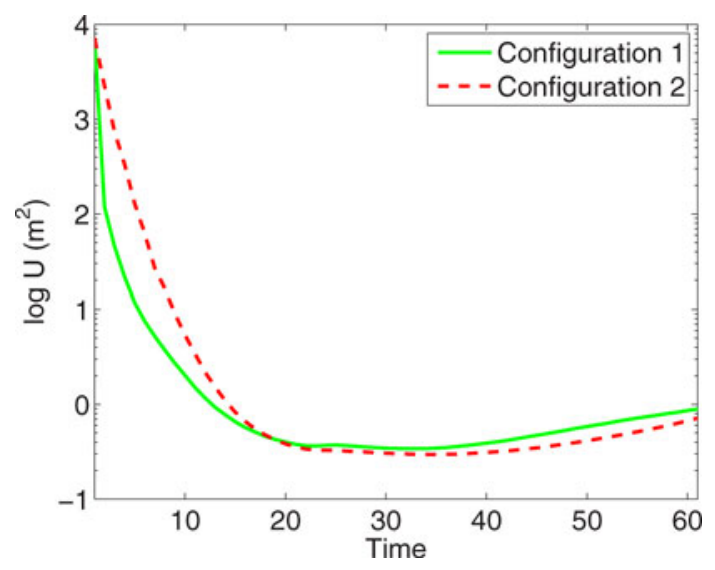

Fig. 6. PCRLB utility as a function of time along the path for the two configurations. The PCRLB predicts that configuration 2 will have difficulty at the beginning of the path.

is superimposed for reference. The path and antenna locations were truthed with a handheld GPS unit.

The utility function (27) plotted in Fig. 2 is the time average of the PCRLB $x / y$ elements for each sensor position. A further breakdown is shown in Fig. 6, which shows the metric as a function of time step. It predicts that configuration 2 will be substantially worse than configuration 1 , primarily at the beginning of the road.

The transmitter was placed at the first location and a dismount walked the path. Then, the transmitter was moved to the second location and the dismount walked the path again. The tracker was executed using the data collected during each collection. The PCRLB utility calculation shown in Fig. 2 predicts that performance with configuration 1 will be much better than with configuration 2 , particularly at the beginning of the vignette (lower right). The tracking results for each configuration, shown in Figs. 7 and 8, indeed show substantially improved tracking performance with the sensor at the PCRLB preferred location.

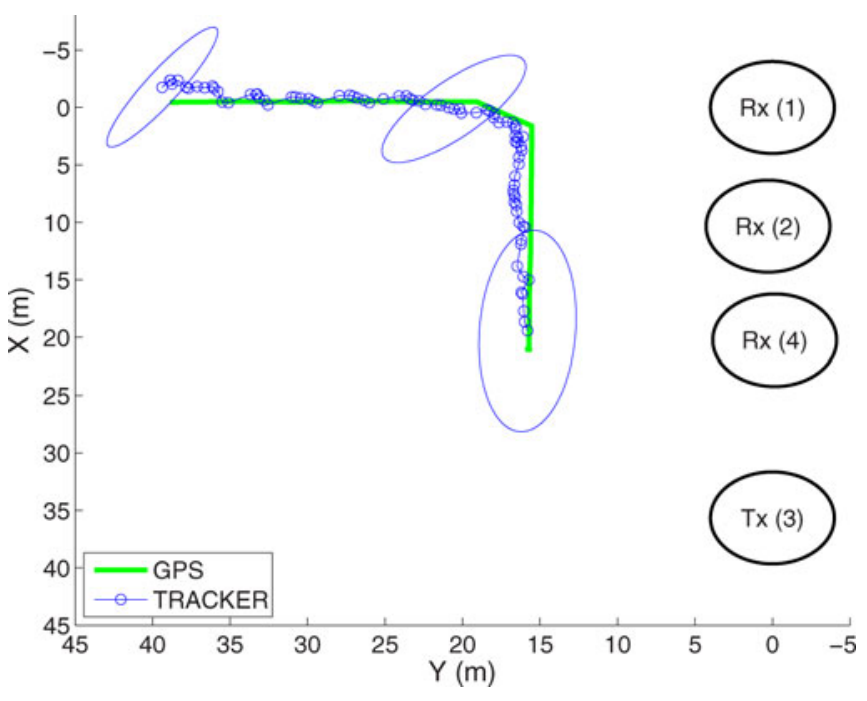

Fig. 7. Tracking results with configuration 1. The tracker estimate is shown in blue, with tracker covariances drawn at selected times. The GPS truth of the path is shown in green. The results show very good agreement between GPS and tracker estimate for this configuration, as predicted by the PCRLB.

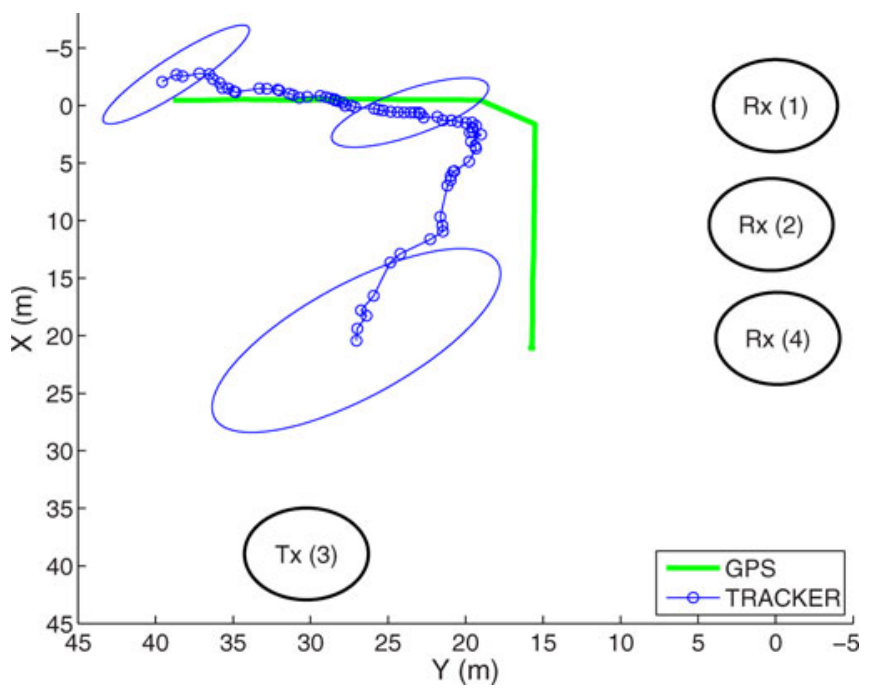

Fig. 8. As predicted by the PCRLB, tracking performance with configuration 2 is much poorer. The main estimation errors occur at the beginning of the path as the PCRLB predicted.

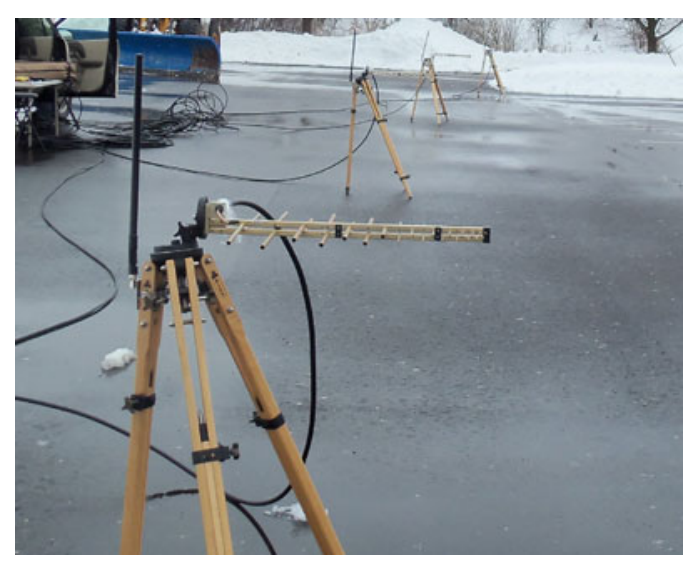

Fig. 9. Four sensors as deployed for the sensor failure experiment. 


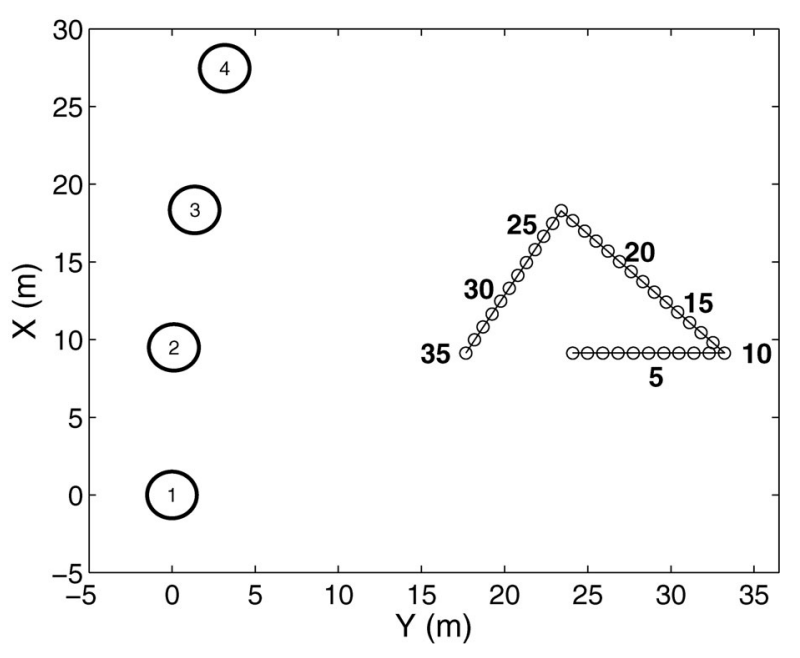

Fig. 10. Collection setup, showing the four sensors and the path. The dismount starts at the bottom of the triangle at time 0 and proceeds in a counterclockwise fashion for $350.5 \mathrm{~s}$ time steps.

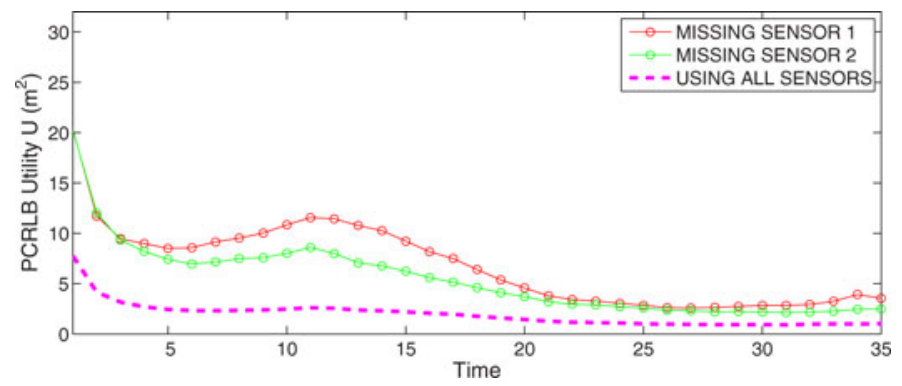

Fig. 11. PCRLB as a function of time computed when all sensors are used, and when either sensor 1 or sensor 2 is missing.

\section{Using the PCRLB to Predict the Effect of Sensor Failure}

This second experiment uses the PCRLB method to predict the effect of a sensor failure. We again deploy four antennas, this time located at $(0,0) \mathrm{m},(9.5,0.1) \mathrm{m},(18.3,1.4) \mathrm{m}$, and $(27.4,3.2) \mathrm{m}$. Each sensor acted as both a transmitter and a receiver, giving 12 bistatic pairs (no monostatic data were collected; therefore, the term $t=r$ is omitted in the FIM calculation). The antenna layout is shown in Fig. 9.

The PCRLB was used to compute the bound on error when individual sensors fail. The prediction was then compared with tracker performance when omitting data from the failed sensor. Data were collected while a dismount moved along a road characterized by the endpoints $(18.3,23.4) \mathrm{m},(9.3,17.7) \mathrm{m}$, and $(9.1,33.2) \mathrm{m}$ at approximately $2 \mathrm{~m} / \mathrm{s}$. Fig. 10 shows the sensor locations and path for this collection.

Again, path and antenna locations were truthed with a handheld GPS. We computed the PCRLB utility as a function of time when using all four sensors (12 bistatic range/range-rate maps at each CPI) using (27). We also computed the bound for the case where antenna 1 was missing and the case where antenna 2 was missing. The computed values as a function of time (position) are shown in Fig. 11.

We compare the bound with tracker mean-square error using measurements that are recorded by the sensors while a dismount

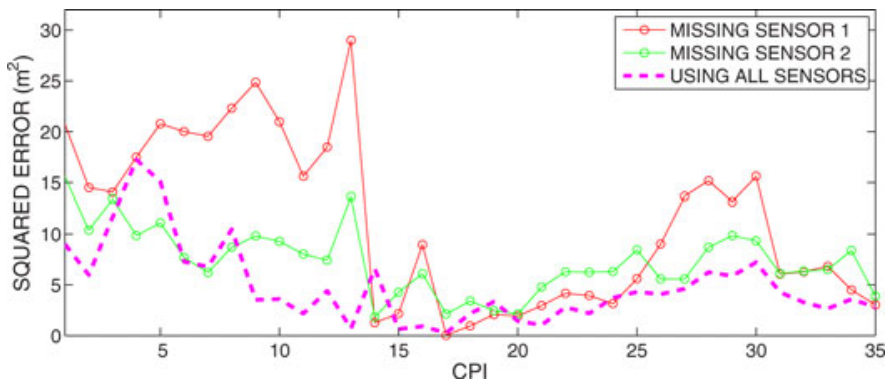

Fig. 12. Observed mean-squared tracking error when all sensors are used and a sensor is omitted. The results are consistent with the bound prediction.

walked the path. Since we collected data from all sensors, we can perform tracking using this full set, and also perform tracking when a sensor is omitted. The mean squared tracking error using data collected by all sensors and cases where either sensor 1 or sensor 2 was missing are given in Fig. 12. The tracking error when sensor 1 is missing is worse than when sensor 2 is missing as predicted. In addition, the tracking error increases around time 13 as predicted by the bound. An interesting future extension to this study may be to model sensor failure when computing the bound, so as to place the constellation to also be robust to sensor failure.

\section{CONCLUSION}

This paper has presented a novel approach to selecting locations for a multistatic RF array. It selects sensor positions to minimize the PCRLB on localization error. One important contribution of this study is the development of a nonGaussian/non-linear sensor model which accurately describes the sensor physics, including transmit and receive beam patterns. A second contribution of this paper is the validation of the technique through a series of field data collected using a constellation of narrowband radar.

\section{APPENDIX A}

\section{PIXELATED RAYleigh MOdEL FiSHER INFORMATION MATRIX}

The $(m, n)$ th element of the FIM $J_{F}(\theta)$ for a sensor model $f(z ; \theta)$ is

$$
\left[J_{F}(\theta)\right]_{m n}=\mathbb{E}_{z}\left[\frac{\partial \ln f(z \mid \theta)}{\partial \theta_{m}} \cdot \frac{\partial \ln f(z \mid \theta)}{\partial \theta_{n}}\right] .
$$

We use a pixelated Rayleigh model, which says that measurements are made in pixels, and the statistical properties of each pixel depend on the true state of the target and the position of the transmitter and the receiver. In particular, for range/Doppler pixel $i, j$, transmitter $t$, and receiver $r$, our model is

$$
f_{i j}\left(z_{i j} ; \theta, t, r\right)=2 \frac{z_{i j}}{\lambda_{i j}^{2}(\theta ; t, r)} \exp \left(-\frac{z_{i j}^{2}}{\lambda_{i j}^{2}(\theta ; t, r)}\right)
$$

where $\lambda_{i j}(\theta ; t, r)$ is the Rayleigh mode that corresponds to $i, j$. Assuming independence across transmitter, receiver, and pixel, 
we have the joint distribution for the measurement $z$ :

$$
f(z ; \theta)=\prod_{t, r} \prod_{i, j} 2 \frac{z_{i j}(t, r)}{\lambda_{i j}^{2}(\theta ; t, r)} \exp \left(-\frac{z_{i j}^{2}(t, r)}{\lambda_{i j}^{2}(\theta ; t, r)}\right) .
$$

The derivation of the FIM with this model is as follows [21]. First, the derivative of the log-likelihood function with respect to the parameter vector $\theta$ is

$$
\begin{aligned}
\frac{\partial \ln f(z ; \theta)}{\partial \theta_{m}}= & 2 \sum_{t, r} \sum_{i, j}\left(\frac{z_{i j}^{2}(t, r)}{\lambda_{i j}^{3}(\theta ; t, r)} \frac{\partial \lambda_{i j}(\theta ; t, r)}{\partial \theta_{m}}\right. \\
& \left.-\frac{1}{\lambda_{i j}(\theta ; t, r)} \frac{\partial \lambda_{i j}(\theta ; t, r)}{\partial \theta_{m}}\right) .
\end{aligned}
$$

Define

$$
y_{i j t r} \doteq \frac{z_{i j}^{2}(t, r)}{\lambda_{i j}^{3}(\theta ; t, r)}
$$

and note that $y$ follows the Gamma distribution, i.e.,

$$
y_{i j t r} \sim \Gamma\left[1, \frac{2}{\lambda_{i j}(\theta ; t, r)}\right]
$$

since we assumed the statistics of $z_{i j}$ are Rayleigh. This yields

$$
\mathbb{E}_{z}\left[y_{i j t r}\right]=\frac{2}{\lambda_{i j}(\theta ; t, r)} \text { and } \mathbb{E}_{z}\left[y_{i j t r}^{2}\right]=\frac{8}{\lambda_{i j}(\theta ; t, r)^{2}}
$$

For notational convenience, let $\lambda_{i j t r}^{\theta} \doteq \lambda_{i j}(\theta ; t, r)$. Therefore, this symbol will indicate the value of the Rayleigh mode parameter in the bistatic range/range-rate cell $i, j$ with a transmitter $t$ and receiver $r$ when the true target state vector is $\theta$.

With this as background, we can then write the FIM directly from (29) as

$$
\begin{aligned}
{\left[J_{F}(\theta)\right]_{m n} } & \\
= & 4 \mathbb{E}_{z}\left[\left(\sum_{t, r} \sum_{i, j} y_{i j t r} \frac{\partial \lambda_{i j t r}^{\theta}}{\partial \theta_{m}}\right)\left(\sum_{p, q} \sum_{u, v} y_{u v p q} \frac{\partial \lambda_{u v p q}^{\theta}}{\partial \theta_{n}}\right)\right. \\
& -\left(\sum_{t, r} \sum_{i, j} y_{i j t r} \frac{\partial \lambda_{i j t r}^{\theta}}{\partial \theta_{m}}\right)\left(\sum_{p, q} \sum_{u, v} \frac{1}{\lambda_{u v p q}^{\theta}} \frac{\partial \lambda_{u v p q}^{\theta}}{\partial \theta_{n}}\right) \\
& -\left(\sum_{t, r} \sum_{i, j} \frac{1}{\lambda_{i j t r}^{\theta}} \frac{\partial \lambda_{i j t r}^{\theta}}{\partial \theta_{m}}\right)\left(\sum_{p, q} \sum_{u, v} y_{u v p q} \frac{\partial \lambda_{u v p q}^{\theta}}{\partial \theta_{n}}\right) \\
+ & \left.\left(\sum_{t, r} \sum_{i, j} \frac{1}{\lambda_{i j t r}^{\theta}} \frac{\partial \lambda_{i j t r}^{\theta}}{\partial \theta_{m}}\right)\left(\sum_{p, q} \sum_{u, v} \frac{1}{\lambda_{u v p q}^{\theta}} \frac{\partial \lambda_{u v p q}^{\theta}}{\partial \theta_{n}}\right)\right] .
\end{aligned}
$$

The only random quantities are $y$. Thus, we can perform the expectations on the second, third, and fourth terms by the straightforward application of (35) and write

$$
\begin{aligned}
& {\left[J_{F}(\theta)\right]_{m n}} \\
& \quad=4 \mathbb{E}_{z}\left[\sum_{t, r} \sum_{i, j} \sum_{p, q} \sum_{u, v} y_{i j t r} y_{u v p q} \frac{\partial \lambda_{i j t r}^{\theta}}{\partial \theta_{m}} \frac{\partial \lambda_{u v p q}^{\theta}}{\partial \theta_{n}}\right]
\end{aligned}
$$

$$
\begin{aligned}
& -4\left(\sum_{t, r} \sum_{i, j} \frac{2}{\lambda_{i j t r}^{\theta}} \frac{\partial \lambda_{i j t r}^{\theta}}{\partial \theta_{m}}\right)\left(\sum_{p, q} \sum_{u, v} \frac{1}{\lambda_{u v p q}^{\theta}} \frac{\partial \lambda_{u v p q}^{\theta}}{\partial \theta_{n}}\right) \\
& -4\left(\sum_{t, r} \sum_{i, j} \frac{1}{\lambda_{i j t r}^{\theta}} \frac{\partial \lambda_{i j t r}^{\theta}}{\partial \theta_{m}}\right)\left(\sum_{p, q} \sum_{u, v} \frac{2}{\lambda_{u v p q}^{\theta}} \frac{\partial \lambda_{u v p q}^{\theta}}{\partial \theta_{n}}\right) \\
& +4\left(\sum_{t, r} \sum_{i, j} \frac{1}{\lambda_{i j t r}^{\theta}} \frac{\partial \lambda_{i j t r}^{\theta}}{\partial \theta_{m}}\right)\left(\sum_{p, q} \sum_{u, v} \frac{1}{\lambda_{u v p q}^{\theta}} \frac{\partial \lambda_{u v p q}^{\theta}}{\partial \theta_{n}}\right) .
\end{aligned}
$$

The last three terms have identical form, simplifying this to

$$
\begin{aligned}
& {\left[J_{F}(\theta)\right]_{m n}} \\
& =4 \mathbb{E}_{z}\left[\sum_{t, r} \sum_{i, j} \sum_{p, q} \sum_{u, v} y_{i j t r} y_{u v p q} \frac{\partial \lambda_{i j t r}^{\theta}}{\partial \theta_{m}} \frac{\partial \lambda_{u v p q}^{\theta}}{\partial \theta_{n}}\right] \\
& \quad-12\left(\sum_{t, r} \sum_{i, j} \frac{1}{\lambda_{i j t r}^{\theta}} \frac{\partial \lambda_{i j t r}^{\theta}}{\partial \theta_{m}}\right)\left(\sum_{p, q} \sum_{u, v} \frac{1}{\lambda_{u v p q}^{\theta}} \frac{\partial \lambda_{u v p q}^{\theta}}{\partial \theta_{n}}\right) .
\end{aligned}
$$

The remaining expectation has independent summands (by assumption) except when $i j=u v$ and $t r=p q$. To simplify, we expand the first term as

$$
\begin{aligned}
& 4 \mathbb{E}_{z}\left[\sum_{t, r} \sum_{i, j} \sum_{p, q} \sum_{u, v} y_{i j t r} y_{u v p q} \frac{\partial \lambda_{i j t r}^{\theta}}{\partial \theta_{m}} \frac{\partial \lambda_{u v p q}^{\theta}}{\partial \theta_{n}}\right] \\
&=4 \sum_{t, r} \sum_{i, j} \sum_{p, q} \sum_{u, v} \mathbb{E}_{z}\left[y_{i j t r}\right] \mathbb{E}_{z}\left[y_{u v p q}\right] \frac{\partial \lambda_{i j t r}^{\theta}}{\partial \theta_{m}} \frac{\partial \lambda_{u v p q}^{\theta}}{\partial \theta_{n}} \\
& \\
& \quad-4 \sum_{t, r} \sum_{i, j} \mathbb{E}_{z}\left[y_{i j t r}\right] \mathbb{E}_{z}\left[y_{i j t r}\right] \frac{\partial \lambda_{i j t r}^{\theta}}{\partial \theta_{m}} \frac{\partial \lambda_{i j t r}^{\theta}}{\partial \theta_{n}} \\
&+4 \sum_{t, r} \sum_{i, j} \mathbb{E}_{z}\left[y_{i j t r} y_{i j t r}\right] \frac{\partial \lambda_{i j t r}^{\theta}}{\partial \theta_{m}} \frac{\partial \lambda_{i j t r}^{\theta}}{\partial \theta_{n}}
\end{aligned}
$$

and then simplify using (35) to

$$
\begin{aligned}
4 \mathbb{E}_{z} & {\left[\sum_{t, r} \sum_{i, j} \sum_{p, q} \sum_{u, v} y_{i j t r} y_{u v p q} \frac{\partial \lambda_{i j t r}^{\theta}}{\partial \theta_{m}} \frac{\partial \lambda_{u v p q}^{\theta}}{\partial \theta_{n}}\right] } \\
= & 4 \sum_{t, r} \sum_{i, j} \sum_{p, q} \sum_{u, v} \frac{4}{\lambda_{i j t r}^{\theta} \lambda_{u v p q}^{\theta}} \frac{\partial \lambda_{i j t r}^{\theta}}{\partial \theta_{m}} \frac{\partial \lambda_{u v p q}^{\theta}}{\partial \theta_{n}} \\
& -4 \sum_{t, r} \sum_{i, j} \frac{4}{\lambda_{i j t r}^{\theta} \lambda_{i j t r}^{\theta}} \frac{\partial \lambda_{i j t r}^{\theta}}{\partial \theta_{m}} \frac{\partial \lambda_{i j t r}^{\theta}}{\partial \theta_{n}} \\
& +4 \sum_{t, r} \sum_{i, j} \frac{8}{\lambda_{i j t r}^{\theta 2}} \frac{\partial \lambda_{i j t r}^{\theta}}{\partial \theta_{m}} \frac{\partial \lambda_{i j t r}^{\theta}}{\partial \theta_{n}}
\end{aligned}
$$

giving finally

$$
4 \mathbb{E}_{z}\left[\sum_{t, r} \sum_{i, j} \sum_{p, q} \sum_{u, v} y_{i j t r} y_{u v p q} \frac{\partial \lambda_{i j t r}^{\theta}}{\partial \theta_{m}} \frac{\partial \lambda_{u v p q}^{\theta}}{\partial \theta_{n}}\right]
$$




$$
\begin{aligned}
= & 16\left(\sum_{t, r} \sum_{i, j} \frac{1}{\lambda_{i j t r}^{\theta}} \frac{\partial \lambda_{i j t r}^{\theta}}{\partial \theta_{m}}\right)\left(\sum_{p, q} \sum_{u, v} \frac{1}{\lambda_{u v p q}^{\theta}} \frac{\partial \lambda_{u v p q}^{\theta}}{\partial \theta_{n}}\right) \\
& +16 \sum_{t, r} \sum_{i, j} \frac{1}{\lambda_{i t r}^{\theta 2}} \frac{\partial \lambda_{i t r}^{\theta}}{\partial \theta_{m}} \frac{\partial \lambda_{i t r}^{\theta}}{\partial \theta_{n}} .
\end{aligned}
$$

Combining this with (38), we have

$$
\begin{aligned}
& {\left[J_{F}(\theta)\right]_{m n}} \\
& =16 \sum_{t, r} \sum_{i, j} \frac{1}{\lambda_{i j t r}^{\theta 2}} \frac{\partial \lambda_{i j t r}^{\theta}}{\partial \theta_{m}} \frac{\partial \lambda_{i j t r}^{\theta}}{\partial \theta_{n}} \\
& \quad+4\left(\sum_{t, r} \sum_{i, j} \frac{1}{\lambda_{i j t r}^{\theta}} \frac{\partial \lambda_{i j t r}^{\theta}}{\partial \theta_{m}}\right)\left(\sum_{p, q} \sum_{u, v} \frac{1}{\lambda_{u v p q}^{\theta}} \frac{\partial \lambda_{u v p q}^{\theta}}{\partial \theta_{n}}\right) .
\end{aligned}
$$

Therefore, the values of the elements in the FIM are the functions of the Rayleigh mode parameter $\lambda_{i j}$ and its derivatives with respect to the target parameter vector $\theta$. The analytic form for these derivatives is given next in Appendix B.

\section{APPENDIX B}

\section{Rayleigh Mode Parameter Model Details}

To completely specify the FIM, we need the derivatives of $\lambda$ given a transmitter located at $\left(x_{T}, y_{T}\right)$ and a receiver located at $\left(x_{R}, y_{R}\right)$. Recall that the Rayleigh mode model from Section II is

$$
\begin{aligned}
\lambda_{i j}(\theta ; t, r)= & \lambda_{B}(t, r) \\
& +\left(\lambda_{T}(t, r)-\lambda_{B}(t, r)\right) \prod_{f=1}^{4} S_{i j}^{f}(\theta ; t, r)
\end{aligned}
$$

where $S^{f}$ are terms that model the SNR suppression from a number of physical factors. Here, we choose to model the transmit beam, the receive beam, direct path energy, SNR range dependence, and the target point spread function.

The derivative of $\lambda$ with respect to the elements of the parameter vector $\theta$ is given from the chain rule as

$$
\begin{aligned}
& \frac{\partial \lambda_{i}(\theta ; t, r)}{\partial \theta_{\circ}} \\
& =\left(\lambda_{T}(t, r)-\lambda_{B}(t, r)\right) \sum_{i=1}^{f} \frac{\partial S_{i j}^{f}(\theta ; t, r)}{\partial \theta_{\circ}} \prod_{\substack{g=1 \\
g \neq f}}^{N} S_{i j}^{g}(\theta ; t, r)
\end{aligned}
$$

and the derivative of the illumination term $S_{i j}^{1}(\theta ; t, r)$ (9) with respect to the elements of the parameter vector $\theta$ is

$$
\begin{aligned}
\frac{\partial}{\partial \theta_{\circ}} S_{i j}^{1}(\theta ; t, r) \\
=2 \operatorname{sinc}\left(c\left(\alpha_{t}-\alpha_{t, \theta}\right)\right) \frac{\partial}{\partial \theta_{\circ}} \alpha_{t, \theta} \\
\quad \times\left[\frac{\cos \left(\pi c\left(\alpha_{t}-\alpha_{t, \theta}\right)\right)}{\alpha_{t}-\alpha_{t, \theta}}-\frac{\sin \left(\pi c\left(\alpha_{t}-\alpha_{t, \theta}\right)\right)}{\pi c\left(\alpha_{t}-\alpha_{t, \theta}\right)^{2}}\right]
\end{aligned}
$$

where

$$
\frac{\partial}{\partial x} \alpha_{t, \theta}=\frac{y_{T}-y}{\left(x-x_{T}\right)^{2}+\left(y-y_{T}\right)^{2}}
$$

and

$$
\frac{\partial}{\partial \dot{x}} \alpha_{t, \theta}=0
$$

The $y$ and $\dot{y}$ derivatives are analogous. The derivative of $S_{i j}^{2}(\theta ; t, r)$ [defined in (10)] is obvious by symmetry. $S_{i j}^{4}(\theta ; t, r)$ [defined in (15)] is independent of $\theta$. The derivative of $S_{i j}^{3}(\theta ; t, r)$ [defined in (14)] is given by

$$
\frac{\partial}{\partial \theta_{\circ}} S_{i j}^{3}(\theta ; t, r)=-2 \Delta_{i j}^{T} A \exp \left(-\Delta_{i j}^{T} A \Delta_{i j}\right) \frac{\partial \Delta_{i j}}{\partial \theta_{\circ}}
$$

with

$$
\frac{\partial \Delta_{i j}}{\partial \theta_{\circ}}=\left(\begin{array}{c}
-\frac{\partial R(\theta ; t, r)}{\partial \theta_{\circ}} \\
-\frac{\partial \dot{R}(\theta ; t, r)}{\partial \theta_{\circ}}
\end{array}\right) .
$$

The needed bistatic range (12) derivatives are

$$
\begin{aligned}
\frac{\partial R(\theta ; t, r)}{\partial x}= & \frac{x-x_{T}}{\sqrt{\left(x-x_{T}\right)^{2}+\left(y-y_{T}\right)^{2}}} \\
& +\frac{x-x_{R}}{\sqrt{\left(x-x_{R}\right)^{2}+\left(y-y_{R}\right)^{2}}}
\end{aligned}
$$

and

$$
\frac{\partial R(\theta ; t, r)}{\partial \dot{x}}=0 .
$$

The $y$ and $\dot{y}$ derivatives are obvious by symmetry. The bistatic range-rate (13) derivatives are

$$
\begin{aligned}
\frac{\partial \dot{R}(\theta ; t, r)}{\partial \dot{x}}= & \frac{x-x_{R}}{\sqrt{\left(x-x_{R}\right)^{2}+\left(y-y_{R}\right)^{2}}} \\
& +\frac{x-x_{T}}{\sqrt{\left(x-x_{T}\right)^{2}+\left(y-y_{T}\right)^{2}}}
\end{aligned}
$$

and

$$
\begin{aligned}
\frac{\partial \dot{R}(\theta ; t, r)}{\partial x}= & \frac{-\dot{y}\left(x-x_{R}\right)\left(y-y_{R}\right)+\dot{x}\left(y-y_{R}\right)^{2}}{\left[\left(x-x_{R}\right)^{2}+\left(y-y_{R}\right)^{2}\right]^{3 / 2}} \\
& +\frac{-\dot{y}\left(x-x_{T}\right)\left(y-y_{T}\right)+\dot{x}\left(y-y_{T}\right)^{2}}{\left[\left(x-x_{T}\right)^{2}+\left(y-y_{T}\right)^{2}\right]^{3 / 2}}
\end{aligned}
$$

The $y$ and $\dot{y}$ derivatives are obvious by symmetry.

\section{ACKNOWLEDGMENT}

The author would like to thank Dr. J. Burns, B. Hart, M. Brennan, and C. Roussi for their help in obtaining the test data used in this study. The author would also like to acknowledge Dr. B. Shapo for helpful discussions about presenting the experiments.

\section{REFERENCES}

[1] B. Himed, H. Bascom, J. Clancy, and M. Wicks, "Tomography of moving targets," in Proc. SPIE-Int. Soc. Opt. Eng., vol. 4540, pp. 608-619, 2001.

[2] L. Lo Monte, R. Ansari, D. Erricolo, and M. C. Wicks, "The use of geometric diversity for spectral dominance in underground imaging," in Proc. 2009 IEEE Wavef. Divers. Design Conf., pp. 55-61. 
[3] R. Tharmarasa, T. Kirubarajan, J. Peng, and T. Lang, "Optimization-based dynamic sensor management for distributed multitarget tracking," IEEE Trans. Syst., Man, Cybern. C, Appl. Rev., vol. 39, no. 5, pp. 534-546, Sep. 2009.

[4] R. Tharmarasa, T. Kirubarajan, and M. L. Hernandez, "Large-scale optimal sensor array management for multitarget tracking," IEEE Trans. Syst., Man, Cybern. C, Appl. Rev., vol. 37, no. 5, pp. 803-814, Sep. 2007.

[5] K. C. Ho and L. M. Vicente, "Sensor allocation for source localization with decoupled range and bearing estimation," IEEE Trans. Signal Process., vol. 56, no. 12, pp. 5773-5789, Dec. 2008.

[6] W. R. Hahn, "Optimum signal processing for passive sonar range and bearing estimation," J. Acoust. Soc. Amer., vol. 58, no. 1, pp. 201-207, Jul. 1975.

[7] G. C. Carter, "Variance bounds for passively locating an acoustic source with a symmetric line array," J. Acoust. Soc. Amer, vol. 62, no. 4, pp. 922 926, Oct. 1977.

[8] J. S. Abel, "Optimal sensor placement for passive source localization," in Proc. IEEE Int. Conf. Acoust., Speech, Signal Process., Apr., 1990, vol. 5, pp. 2927-2930.

[9] O. Erdinc, P. Willett, and S. Coraluppi, "Multistatic Sensor Placement: A Tracking Approach," J. Adv. Inf. Fusion, vol. 2, no. 1, pp. 22-34, Jul. 2007.

[10] S. Coraluppi, "Multistatic sonar localization," IEEE J. Ocean. Eng., vol. 31, no. 4, pp. 964-974, Oct. 2006.

[11] M. L. Hernandez, T. Kirubarajan, and Y. Bar-Shalom, "Multisensor resource deployment in the presence of clutter using Cramér-Rao lower Bounds," IEEE Trans. Aerosp. Electron. Syst., vol. 2, no. 40, pp. 399416, Apr. 2004

[12] M. Chernaikov, Bistatic Radar. West Sussex, UK, Wiley, 2007.

[13] J. C. Toomay and P. J. Hannen, Radar Principles, 3rd ed. Norwich, NY, Scitech, 2004

[14] M. Skolnik, Radar Handbook. Boston, MA: McGraw-Hill, 1990.

[15] H. Van Trees, Detection, Estimation, and Modulation Theory, Part I. New York: Wiley, 1968.

[16] B. Z. Bobrovsky, E. Mayer-Wolf, and M. Zakai, "Some classes of global Cramér-Rao bounds," Ann. Stat., vol. 15, pp. 1421-1438, 1987.

[17] A. Weiss and E. Weinstein, "A lower bound on the mean square error in random parameter estimation," IEEE Trans. Inf. Theory, vol. 31, no. 5, pp. 680-682, Sep. 1985.

[18] T. Sathyan, M. L. Hernandez, A. Sinha, and T. Kirubarajan, "WeissWeinstein lower bound for maneuvering target tracking," Proc. SPIE, vol. 6236, pp. 62360G, 2006

[19] A. J. Weiss, "Fundamental Bounds in Parameter Estimation," Ph.D. dissertation, Tel-Aviv Univ., Tel-Aviv, Israel, 1985.

[20] H. Van Trees, Bayesian Bounds for Parameter Estimation and Nonlinear Filtering/Tracking. Piscataway, NJ, Wiley-IEEE Press, 2007.

[21] C. Kreucher, J. W. Stayman, B. Shapo, and M. Stuff, "Exploiting a constellation of narrow band RF sensors to detect and track moving targets," in Proc. Asilomar Conf. Signals, Syst. Comput., Nov. 7-10, 2010, pp. 1966-1970.
[22] P. Tichavsky, C. Muravchik, and A. Nehorai, "Osterior Cramér-Rao bounds for discrete-time nonlinear filtering," IEEE Trans. Signal Process., vol. 46, no. 5, pp. 1386-1396, May 1998.

[23] K. Kastella and C. Kreucher, "Multiple model nonlinear filtering for low signal ground target applications," IEEE Trans. Aerosp. Electron. Syst., vol. 41, no. 2, pp. 549-564, Apr. 2005.

[24] Z. Kassas and U. Ozguner, "A nonlinear filter coupled with hospitability and synthetic inclination maps for in-surveillance and out-of-surveillance tracking," IEEE Trans. Syst., Man, Cybern. C, Appl. Rev., vol. 40, no. 1, pp. 87-97, Jan. 2010.

[25] S. Yang and S. Jat, "Genetic algorithms with guided and local search strategies for university course timetabling," IEEE Trans. Syst., Man, Cybern. C, Appl. Rev., vol. 41, no. 1, pp. 93-106, Jan. 2011.

[26] Z. Z.-H. Zhan, Z. Jun, L. Yun, and H. S.-H Chung, H.S.-H., "Adaptive Particle Swarm Optimization," IEEE Trans. Syst., Man, Cybern. B: Cybern. vol. 39, no. 6, pp. 1362-1381, Dec. 2009.

[27] Y. S. Ong, N. Krasnogor, and H. Ishibuchi, "Special issue on memetic algorithms," IEEE Trans. Syst., Man, Cybern. B, Cybern., vol. 37, no. 1 , pp. 2-5, Feb. 2007.

[28] J. Gallardo, C. Cotta, and A. Fernandez, "On the hybridization of memetic algorithms with branch-and-bound techniques," IEEE Trans. Syst., Man, Cybern. B, Cybern., vol. 37, no. 1, pp. 77-83, Feb. 2007.

[29] H. Topcuogly, M. Ermis, and M. Sifyan, "Positioning and utilizing sensors on a 3-D terrain: Part I. Solving with a hybrid evolutionary algorithm," IEEE Trans. Syst., Man, Cybern. C, Appl. Rev., vol. 41, no. 4, pp. 470-480, Jul. 2011.

[30] W. Lipo, L. Sa, F. Tian, and F. Xiuju, "A noisy chaotic neural network for solving combinatorial optimization problems: Stochastic chaotic simulated annealing," IEEE Trans. Syst., Man, Cybern. B, Cybern., vol. 34, no. 5 , pp. 2119-2125, Oct. 2004.

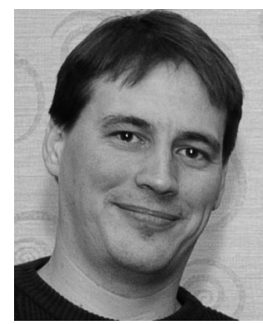

Chris Kreucher received the Ph.D. degree from the University of Michigan, Ann Arbor, in electrical engineering for a dissertation titled "An InformationTheoretic Approach to Sensor Management" in 2005.

From 1998 to 2007, he was a member of the research staff at General Dynamics Corp. (formerly Environmental Research Institute of Michigan) in Ann Arbor, Michigan. Since 2008, he has been with Integrity Applications Inc., Ann Arbor, where he is a Principal System Engineer. His recent research interests include detection, tracking, multisensor fusion, 